\title{
Circuit
}

Musiques contemporaines

\section{Entendu dans Cette ville étrange}

\section{L'un de nous}

\section{Michel Gonneville}

Volume 22, numéro 1, 2012

URI : https://id.erudit.org/iderudit/1008974ar

DOI : https://doi.org/10.7202/1008974ar

Aller au sommaire du numéro

Éditeur(s)

Les Presses de l’Université de Montréal

ISSN

1183-1693 (imprimé)

1488-9692 (numérique)

Découvrir la revue

Citer ce compte rendu

Gonneville, M. (2012). Compte rendu de [Entendu dans Cette ville étrange : l'un de nous]. Circuit, 22(1), 103-108. https://doi.org/10.7202/1008974ar

Ce document est protégé par la loi sur le droit d'auteur. L'utilisation des services d'Érudit (y compris la reproduction) est assujettie à sa politique d'utilisation que vous pouvez consulter en ligne.

https://apropos.erudit.org/fr/usagers/politique-dutilisation/
Cet article est diffusé et préservé par Érudit.

Érudit est un consortium interuniversitaire sans but lucratif composé de l’Université de Montréal, l'Université Laval et l'Université du Québec à Montréal. Il a pour mission la promotion et la valorisation de la recherche. https://www.erudit.org/fr/ 


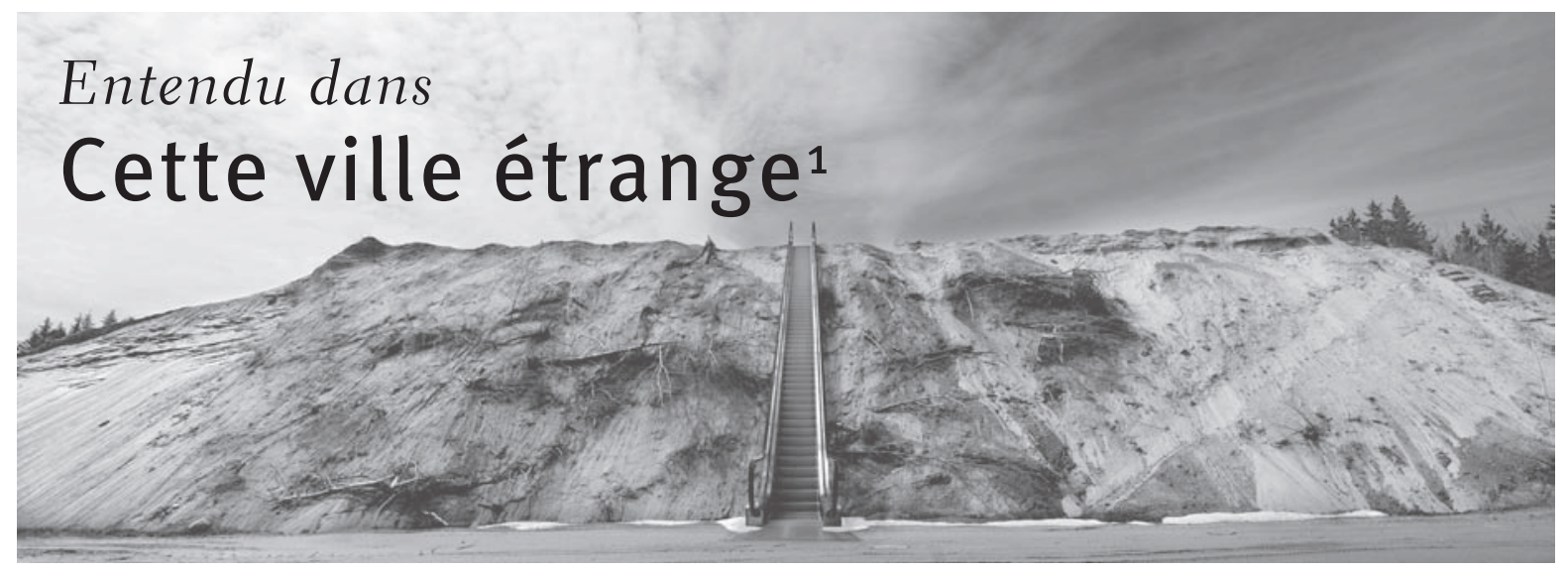

\section{L'un de nous'}

Julien Bilodeau a l'heur d'être encore relativement jeune, et non un «cacique de l'establishment universitaire», et les deux pièces pour orchestre que le critique du Devoir, Christophe Huss, a entendues de lui le rendent aux yeux de ce dernier «aussi prometteur qu'un Guillaume Connesson en France » (Le Devoir, 7 septembre 2011).

Malaise : les extraits de la musique du compositeur français, que l'on peut entendre sur le site internet de ce dernier, montrent un habile talent, en effet, mais qui le situerait plutôt dans la lignée du prolifique compositeur de musique de film John Williams (Jaws, Star Wars, Raiders of the Lost Ark, E.T., Harry Potter, etc.) , avec des titres évoquant aussi souvent la filiation que le style musical lui-même (Trilogie cosmique, Constellations, The Shining One, etc.). Malgré ses accents «classiques », il me semble que la pièce Qu'un cri élève nos chants!, commandée à Bilodeau par l'Orchestre symphonique de Montréal pour l'inauguration de la Maison symphonique, proposait à l'auditeur quelques péripéties d'une autre qualité...

De toute évidence, en rapatriant Bilodeau dans la même famille que Connesson, le critique témoigne surtout de sa méconnaissance des pièces récentes du compositeur québécois, essentielles pour comprendre une démarche intrigante. Car il s'agit de musiques qui plongeraient probablement ledit critique dans la perplexité... Cette forme de confusion de la part de Christophe Huss est au service d'une entreprise de récupération, qui semble prendre sa source dans un conflit jamais réglé avec le père (Boulez...): il s'agit d'abord et avant tout de valoriser une tendance en en dénigrant d'autres qu'il serait trop exigeant d'interroger pour les comprendre. Il s'agit, oui, de ne
1. Chronique sur l'actualité de la création musicale réalisée en collaboration avec les éditeurs du site internet cettevilleétrange.org, soit Julien Bilodeau, Michel Gonneville et Patrick Saint-Denis. Des prolongements de cette collaboration sont publiés sur le site (voir www.cettevilleetrange.org). Les lecteurs sont invités à réagir en écrivant à info@revuecircuit.ca ou à proposer une publication en écrivant à info@ cettevilleetrange.org. Photo: David Champagne.

2. Cet article a pour point de départ l'audition de l'œuvre Qu'un cri élève nos chants! par Julien Bilodeau, commande de l'Orchestre symphonique de Montréal créée par l'OSM sous la direction de Kent Nagano, le 7 septembre 2011 à La Maison symphonique à Montréal. 
3. Cette influence d'une activité analytique approfondie sur la genèse de certains traits du style d'un compositeur se retrouve aussi chez Vivier. En se rappelant qu'il a analysé Arcana dans le cadre de son Concours en analyse au Conservatoire de musique de Montréal, on fera le lien entre cette œuvre et les mélodies quasi incantatoires de Vivier, s'articulant sur un nombre très limité de notes.

4. Il y a une anecdote savoureuse à propos du compositeur Mauricio Kagel qui, indigné à l'audition de cette harmonisation de la mélodie d'Inori, aurait rageusement stoppé le magnétophone et ainsi cassé la bande de l'enregistrement qu'on lui avait prêtée...

5. Voir Alessandro Baricco (1998), L'âme de Hegel et les vaches du Wisconsin, Paris, Albin Michel. pas trop s'éloigner du familier, du connu, d'une certaine façon de concevoir le musical...

La commande faite à Julien Bilodeau devait entretenir des liens avec la Neuvième Symphonie de Beethoven, pièce de résistance du concert. Défi relevé: quelques éléments-signatures de chaque mouvement de la Neuvième sont souvent clairement identifiables et subtilement mêlés à l'aventure formelle (quinte initiale du premier mouvement, rythme principal du Scherzo, accord initial du quatrième mouvement, etc.). Par ailleurs, l'écriture motivique et rythmique (cet ostinato des cordes qui soutient de nombreuses sections), les combinaisons orchestrales très habiles (cela sonne!), et même la menée formelle dramaturgique, quasi expressionniste, de l'œuvre de Bilodeau pourraient paraître presque «classiques» aux oreilles de certains, voire provoquer un malaise chez les tenants d'une approche plus pure et dure, résolument innovatrice (ou «moderne») de la composition contemporaine. Pourtant, si l'on se rappelle que Bilodeau a rédigé au Conservatoire un mémoire d'analyse sur Inori de Stockhausen, certains traits chromatiques en expansion, certains développements contrapuntiques et certaines approches de climax peuvent clairement s'entendre comme issus de passages similaires que l'on entend dans la dernière partie, Polyphonie, de l'œuvre du maître allemand 3 .

Il est intéressant de faire un autre lien entre cette œuvre atypique, unique en son genre, et le malaise évoqué plus haut. Inori a aussi été reçue de façon ambiguë: si on y trouve de remarquables inventions techniques (tempi chromatiques perceptibles, orchestration intimement liée aux intensités), des audaces formelles étonnantes (les 25 premières minutes de cette pièce d'une heure se déroulent autour d'une seule note), il y a aussi des propositions qui, par leur côté «rétrospectif», étaient plutôt déroutantes pour l'époque (retour à un thématisme mélodique clair), voire encore aujourd'hui (harmonisation quasi-hollywoodienne de cette mélodie4, contrepoint quasi-classique de la mélodie se superposant à des transformations d'elle-même).

Pour fonder son propre style, Bilodeau n'aurait-il retenu de son étude d'Inori que les traits de langage les plus «accessibles», les mieux ancrées dans les habitudes d'écoute? Élargissons la question : le cas échéant, Bilodeau estil devenu, ce faisant, complice d'un système de la «musique classique» (la fameuse «musique cultivée » d'Alessandro Barrico5) en offrant à l'auditorat frileux des concerts symphoniques de quoi se rincer l'oreille sans que cette dernière ne frise trop? On soupire en considérant les nombreux compromis auxquels le système de l'orchestre symphonique hérité du XIXe siècle force le compositeur, tout comme le mélomane: programmes de sempiternels 
classiques, place et durée restreintes des œuvres modernes (ici: québécoises), décorum, langage, traitement et image médiatiques (télé, radio, journaux, critiques) plus que douteux et parfois affectés... Faut-il que le compositeur joue le jeu d'un appareil qui le dépasse et dont il pourrait pourtant, en son for intérieur, réprouver de nombreux aspects?

Attachons-nous à quelques œuvres récentes de Bilodeau ${ }^{6}$. Ici, on trouvera à la fois des propositions beaucoup plus radicales («modernes») que celles de la pièce pour orchestre, mais aussi des alliages composites parfois inattendus sur le plan stylistique. Dans sa Foucade (2009-2010) pour piano et électronique, Bilodeau, parti (encore une fois) d'un modèle stockhausenien (Mantra), réussit un mariage singulier entre d'une part, une écriture pianistique curieusement «années 1960 » sur les plans du geste et de l'harmonie, d'une virtuosité parfois presque free jazz, et d'autre part, une approche du traitement électronique qui renouvelle le modèle par sa souplesse et sa variété. Dans Kroniks-01 (2005) pour flûte et électronique, il associe une écriture instrumentale et un traitement électronique ancrés dans les techniques de la décennie à un contrôle harmonique presque classique (malgré ses ruptures) et surtout à une surprenante mélodie terminale, lyrique, impressionniste, le parcours formel absorbant le tout, finalement, en une dramaturgie convaincante et expressive.

Le centre de [INKS] (2008) pour ensemble de chambre est une lente progression (chromatique) ascendante, une sorte d'adagio souvent doux mais tendu, où certaines harmonies octaviantes ou consonantes ne déparent d'aucune connotation incongrue un propos général caractérisé principalement par une rythmique floue et une articulation de type «statistique » (nuages de sons sur harmonies statiques).

Mais on retrouvera dans les pièces pour plus grands ensembles - effet du genre sur le style? -, soit dans À coups (2004) écrite pour les 15 musiciens du Nouvel Ensemble Moderne et dans Myriades (2004), commande de l'Orchestre de la Francophonie canadienne, de nombreux «gestes »- une agogique - plus historiquement connotés: dans la première pièce, voici des accents jazz avec ponctuations de batterie, des développements de cellules à la Stravinsky (Le Sacre), une dramaturgie faite de montées, climax et désinences, avec ruptures parfois étonnantes; dans la seconde, outre certains des éléments déjà cités, voilà aux cordes un contrepoint adagio presque bartokien, des harmonies «célestes» accompagnant un solo de piccolo, une «chevauchée » endiablée soutenant des sonneries de trompettes et des interventions de cuivres à la Sibelius.
6. Remerciements au compositeur pour les enregistrements gracieusement fournis. 
On se retrouve alors tout près du style de Qu'un cri élève nos chants!... Où est donc Bilodeau dans tout cela? En jouant sur deux tableaux: d'une part, musique plus près de certains acquis historiques de l'oreille (pour «plaire»?) et d'autre part, musique «de recherche» plus subtile et sophistiquée, Bilodeau suit-il un curieux chemin éthique, quasi machiavélique?

En comparant Qu'un cri... avec ces quelques autres œuvres de Bilodeau que nous avons évoquées, la perplexité ne pouvait donc que se creuser... À croire que le compositeur québécois a aussi hérité de Stockhausen ce désir de ne jamais se répéter, de toujours se renouveler, même sur le plan du langage et du style (!), ce qui est évidemment déroutant pour un auditeur qui recherche les traits reconnaissables d'une démarche. Ou alors, Bilodeau, encore jeune, en est actuellement à se constituer une panoplie de techniques au fur et à mesure des projets. Pour l'OSM, il a peut-être choisi de mettre la pédale douce à quelques désirs profonds plus prospectifs pour répondre à certaines attentes, que ces attentes aient été explicites de la part du commanditaire ou introjectées par le compositeur lui-même. Mais si Kent Nagano cherchait «un son» (dixit le compositeur), fallait-il nécessairement y pourvoir?... Pacte avec le diable? Compromission? Le jeune homme qui est passé par certaines institutions de la musique contemporaine (Stockhausen, Nouvel Ensemble Moderne, Ensemble Moderne Frankfurt, Ircam, etc.) at-il renié son tout récent passé, ses ambitions, ses goûts?

On l'aura perçu: les interrogations lancinantes qui précèdent renvoient aux vieilles discussions sur ce qui est admissible ou non sur le plan esthétique, sur ce qui apporte une contribution à l'évolution historique de la musique - selon une lecture de l'Histoire musicale et artistique souvent trop pressée à identifier «ce qui compte» parmi les nombreuses et diversifiées propositions des créateurs musicaux, une lecture où se sent encore le vieux réflexe de lancer des anathèmes ou de décréter des exclusives... Les attentes quant à la cohérence de la démarche d'un artiste font partie de ce débat, que le pluralisme actuel n'a pas réussi à amortir complètement. Ce genre de discussion, tournant souvent «à l'interne», doit être replacé dans un contexte plus large.

Les nombreuses contradictions du système sociologique de la musique (et de l'art), mises en évidence par Adorno, Menger et d'autres, ne sont rien pour apaiser les interrogations d'un créateur quant à sa propre fonction. Aux côtés du compositeur-clown, amuseur public, entertainer, tâcheron, serviteur parmi les serviteurs, habile artisan, pourvoyeur de musique pour le plaisir de l'Évêque, du Roy ou du public bourgeois, qui en quelque sorte conforte et 
flatte toute une organisation politico-économique dont les méfaits ne sont plus à rappeler; aux côtés du créateur messianique, ultime porteur de la transcendance et de l'idéal, génie travaillant dans la solitude pour l'avènement espéré de l'Homme illuminé, qui saura enfin gérer le monde dans une harmonie respectueuse; aux côtés du concepteur structuraliste, centré sur les pures nécessités de l'Art pour l'Art; aux côtés du témoin engagé, dont les propositions critiques veulent révéler et dénoncer les contradictions et horreurs de notre monde; aux côtés de l'observateur ironique de la machine socioartistique; au-delà de toutes ces fonctions que l'artiste a endossées et que lui ont assignées le Moyen-Âge, la Renaissance, le baroque, le classicisme, le romantisme, le modernisme et le post-modernisme, quoi? n'y aurait-il pas de la place pour une autre vision du créateur musical, peut-être plus fondamentale et intégratrice?

Plus essentiellement, il me semble, l'artiste devrait nous apparaitre d'abord et avant tout comme l'un de nous, comme un être humain étonné de sa propre existence et de la société dans laquelle il vit, voire de l'Art même qu'il exerce et à l'évolution duquel il cherche à contribuer. Manipulateur, à chaque fois vierge, de la matière (sonore, visuelle, textuelle) posée devant lui, il peut faire sourire, réfléchir ou encore provoquer ses congénères, libre, comme on le lui souhaite, de les consoler ou de les terrifier, subtilement ou sans égards, avec des moyens développés selon les nécessités, toujours autres. Libre, oui, de les divertir et distraire..., termes dont on ne devrait peut-être pas rougir trop vite, en cédant aux connotations négatives dont on voudrait les charger plutôt que de retourner à leur étymologie (di-vertir, dis-traire...). Faut-il encore redire la nécessité de l'évasion, du rêve, de l'imaginaire? Ultimement - car il s'agit bien de cela -, l'artiste est cet homme ou cette femme libre de faire «acte de communication ", de faire un geste à l'endroit de «l'autre », en espérant que cet autre accepte de sortir de lui-même, librement lui aussi, pour se soumettre à «l'épreuve d'une rencontre »7.

Dans cette perspective plus large, si l'Art est dans la manière de dire, de faire, elle-même tributaire des moyens choisis, peut-on encore blâmer l'artiste de se constituer un coffre à outils étendu, dans le cadre d'une démarche menée à la fois «par volonté et par hasard ${ }^{8}$ ", et parfois aussi par realpolitik? Une démarche à laquelle, ultimement, la Mort seule viendra mettre un terme, fermant le corpus laissé en héritage, dont la musicologie se chargera éventuellement de démêler les fils et de rabibocher une cohérence.
7. Expressions tirées de George Steiner (1991), Réelles présences, Paris, Gallimard, p. 170

8. Titre d'un recueil d'entretiens entre Pierre Boulez et Célestin Deliège (1975), Paris, Seuil. 
9. De plus, je serais bien mal venu de lancer quelque pierre à Bilodeau, moi qui compose périodiquement l'une ou l'autre chanson pour des occasions privées, qui me suis hasardé au dialogue des styles et qui viens de consacrer plusieurs mois à composer un conte musical dans lequel mélodie, modalisme, rythmes vont fouiller dans un canon personnel où se mêlent, justement, chanson et musique rock progressiste...

10. À paraître sur www.cettevilleetrange. org
Avec ces belles propositions trop générales, ne suis-je pas en train de tomber dans l'hérésie de l'éclectisme, où «tout se vaut», et, par la bande, d'essayer de «pardonner» à Julien Bilodeau d'avoir pratiqué l'art du compromis? Ici pourtant, rien de comparable avec les solutions d'un Chostakovitch. Dans un examen plus attentif de l'œuvre composée pour l'OSM, en vue d'en interpréter l'aventure formelle, d'en déchiffrer l'un des «sens » possibles, la fin serait peut-être la clé: à la place d'une ironie grinçante comme chez le Russe, au lieu du cataclysme ou de l'apothéose romantique, plutôt une série de points de suspension, probablement plus modernes et critiques qu'il n'y paraît, et qui semblent dire: «La suite nous appartient... Laissons au Beethoven romantique la «naïveté» de la foi en la fraternité. Quant à nous, oui, essayons d'être moins cruels les uns envers les autres, mais en assumant cette distance congénitale entre nous, qui nous sépare essentiellement, nous pourtant si semblables.»

Je suis certain que Julien Bilodeau assume chacune des notes qu'il a écrites. Et je dois aussi, au terme de ces réflexions, avouer que chacune des pièces qu'il a commises et que j'ai pu entendre m'a parlé, chacune à sa manière9. Est-ce du «relativisme mou» que de ne pas chercher dans une proposition artistique autre chose que ce qu'elle offre?...

Enfin, pour boucler une autre boucle, l'exercice auquel je me suis livré aura peut-être réussi, au moins, à démontrer qu'il était injustifiable de tenter d'assimiler la démarche de ce compositeur à un simple copinage stylistique avec Guillaume Connesson...

Cette pérégrination m’a mené au seuil d'une porte, où j’ai frappé... Une réponse est promise ${ }^{10}$.

\section{Michel Gonneville} 22 octobre 2011

P.S. : Il faut bien vivre... Plus pragmatiquement, et comme un autre contresujet au thème proposé par Christophe Huss, je voudrais souhaiter à Julien Bilodeau et à tous les jeunes créateurs de sa génération une liberté artistique et financière (grâce à des postes de futurs «caciques »?...) qui permette, avec un contexte social plus accueillant et audacieux, l'expression de leur plein talent, pour notre plaisir, édification, stupeur, étonnement, émotion, fierté, etc. 